\title{
Extension of the First Spectrum of Rhodium $(\mathrm{Rh} \mathrm{I})^{1}$
}

\author{
Robert J. Murphy
}

\begin{abstract}
The arc spectrum of rhodium has been investigated photographically in the region 6300 to $12000 \mathrm{~A}$. The spectrum was excited in conventional direct-current ares and photographed on infrared sensitive emulsions with the aid of concave diffraction gratings. Wavelengths have been measured for $238 \mathrm{Rh}$ r lines, including 90 new lines, of which 53 have been classified. Two new even levels have been added to the list of known term values; these two new levels account for 18 of the 53 newly classified $\mathrm{Rh}$ I lines.
\end{abstract}

\section{Introduction}

The analysis of the RhI spectrum has been extended to the long-wave limit of photographic emulsion sensitivity, in order to provide additional term values of Rh I for volume III of Bureau Circular 467, now being compiled [1]. ${ }^{2}$

Except for studies of Zeeman effect, no investigations of this spectrum have been made since 1925 , when Meggers [2] published a paper on the platinum metals. That paper gave the wavelengths of 551 rhodium lines between 4504 and 8615 A. In 1901 C. P. Snyder [3] published "The wave-number system of rhodium", containing regularities among $476 \mathrm{Rh}$ I lines. The significance of these regularities was not realized until 25 years later, when a partial interpretation of this system was given by Meggers and Kiess [4].

Prior to 1940 the only analysis of the Zeeman effect of rhodium was that given by Sommer [5] in 1927. He lists 136 atomic energy levels and classifies approximately 1,000 lines, about 90 of which are accompanied by data on the Zeeman effect. In 1940 an analysis by Molnar and Hitchcock [6] improved and extended the earlier work of Sommer [5]. They list wavelengths, relative intensities, and vacuum wave numbers (both observed and computed) for 446 lines from 1988 to $8615 \mathrm{~A}$, in addition to 29 terms, and $g$-values for 108 levels. The term values from their paper have been used in the present investigation.

Thus the extension of the description and analysis of Rh r from 8615 to $11021 \mathrm{~A}$ is desirable and has been made possible mainly by the use of the new photographic emulsions now available for spectroscopic studies. These emulsions are sensitive much farther into the infrared than they were in 1925 . This is illustrated by the thallium line at 13013 A recently photographed [7] in the Bureau's Spectroscopy Laboratory, which sets a new mark for long-wave photography of laboratory arc sources.

\section{Experimental Details}

The spectrum, RhI, as presented in this paper was excited by means of direct-current ares between electrodes of metallic rhodium. These electrodes,

1 This paper was submitted to Boston College in partial fulfillment of the requirements for the Master of Science degree in May 1952.

2 Figures in brackets indicate literature references at the end of this paper. measuring approximately 6 by $10 \mathrm{~mm}$, were made by pressing rhodium-metal powder in a Dietert hydraulic press. These solid cylinders of rhodium were pinched in slotted copper rods that served as electrode holders. The power was drawn from a $220 \mathrm{v}$ d-c circuit, with currents of 6 to 8 amp.

All the spectrograms in this investigation were made with concave diffraction gratings set up in Wadsworth mountings, as described by Meggers and Burns [8].

The range from 6300 to $8700 \mathrm{~A}$ was photographed with Eastman I-N plates, from 8000 to 10200 A with Eastman I-Q plates, and from 9700 to $12000 \mathrm{~A}$ with Eastman I-Z plates. The I-Q and I-Z plates were hypersensitized according to the method described by Burka [9]. This consisted of bathing the plates for 2 min in 2- to 6-percent solutions of 28-percent ammonia, followed by a 2-min wash in alcohol and rapid drying with warm air.

For the N-sensitivity region, a 15,000 lines/in. grating was used. The first-order spectrum of this grating has a reciprocal dispersion of $4.85 \mathrm{~A} / \mathrm{mm}$. The long-wave $Z$-sensitivity region was photographed in the first order of a 7500 lines/in. grating. In its first-order spectrum this spectrograph has a reeiprocal dispersion of $10 \mathrm{~A} / \mathrm{mm}$. The $\mathrm{Q}$-sensitivity region lying between the $\mathrm{N}$ - and $\mathrm{Z}$-sensitivity regions, was investigated with both gratings described above.

Exposures varied from $20 \mathrm{~min}$ in the $\mathrm{N}$ region to $4 \mathrm{hr}$ and $7 \mathrm{hr}$, respectively, in the $\mathrm{Q}$ and $\mathrm{Z}$ regions. In the long-wave region, a dark-red-glass filter placed in front of the spectrograph slit eliminated second and higher overlapping spectral orders and permitted the first-order Rh I spectrum to be photographed. For shorter wavelengths, a yellow-glass filter was used to give the first-order spectrum of rhodium.

For the spectral ranges covered by the I-Z and I-Q plates, iron wavelength standards adopted by the International Astronomical Union [10] were taken from the second- or third-order spectra and converted to first order, but for the range observed with I-N plates the iron comparison spectrum was recorded in the same first order as the rhodium spectrum.

\section{Results}

Measurements of the line positions for wavelength determinations, and visual estimates of line intensities and character, were made on a Gaertner 
moving-carriage comparator. This instrument gives readings directly to $1 \mu$. Each $R h_{I}$ line retained in the final list was observed and measured at least three times, and many were remeasured several times to increase wavelength accuracy. The probable error for most wavelengths is approximately $0.02 \mathrm{~A}$. Toward the long wavelengths, where most of the lines were recorded as hazy, which makes an accurate comparator setting quite difficult, the error approaches $0.05 \mathrm{~A}$. The range of estimated relative intensities is from " 1 ?", which indicates a weak line difficult to bisect, to " 800 " for the strongest $\mathrm{Rh}$ I line.

The final values of wavelengths, estimated relative intensities and line character, vacuum wave numbers, and term combinations of classified lines are given in table 1. For classified lines, the wave number calculated from the energy levels is also given. The term notation is that used by Molnar and Hitchcock [6].

TABLE 1. Extension of the first spectrum of rhodium, Rh I

$[h=$ hazy; $H=$ very hazy; $w=$ wide; $W=$ very wide; $d=$ double; $l=$ shaded to longer waves; $s=$ shaded to shorter waves; ?=observation doubtful $]$

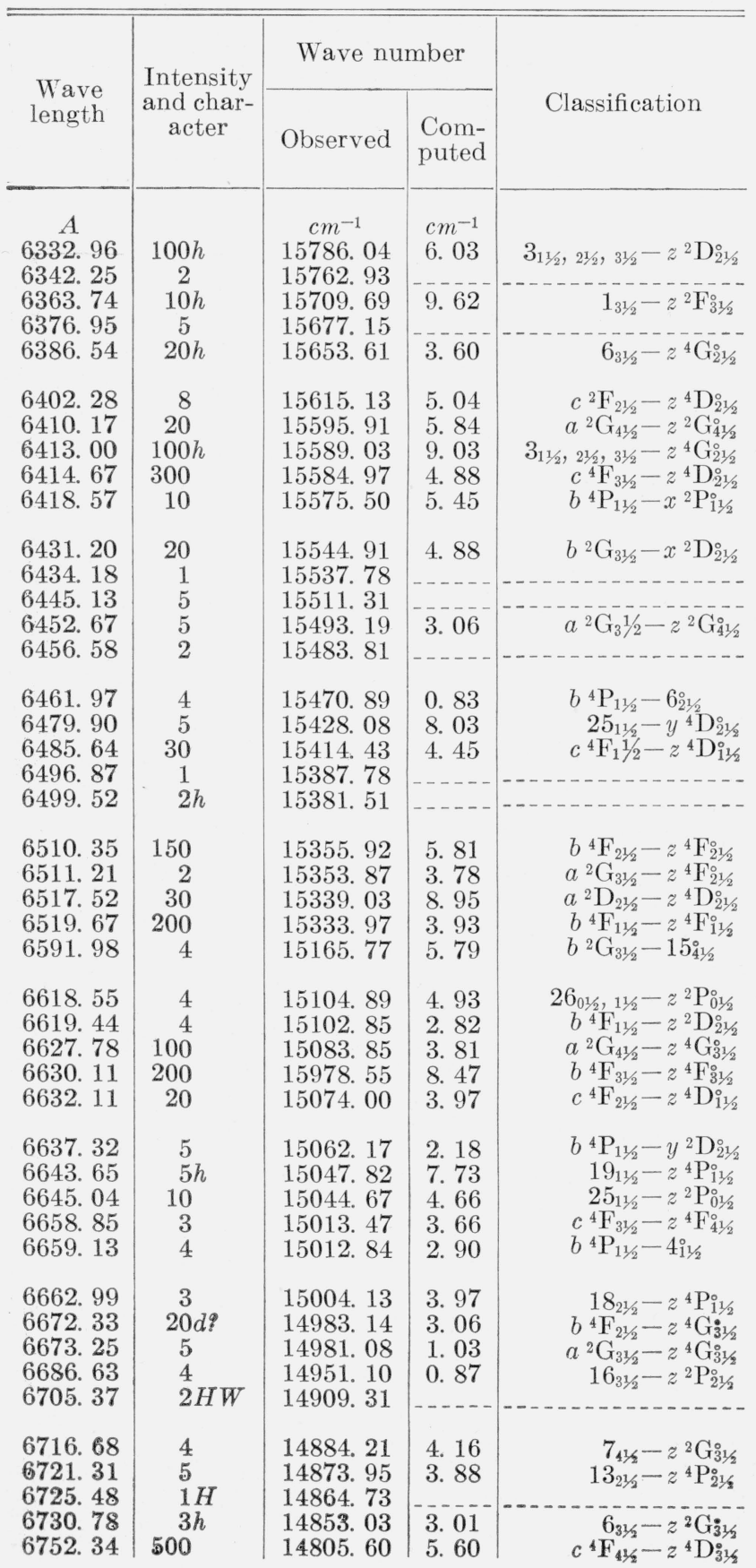

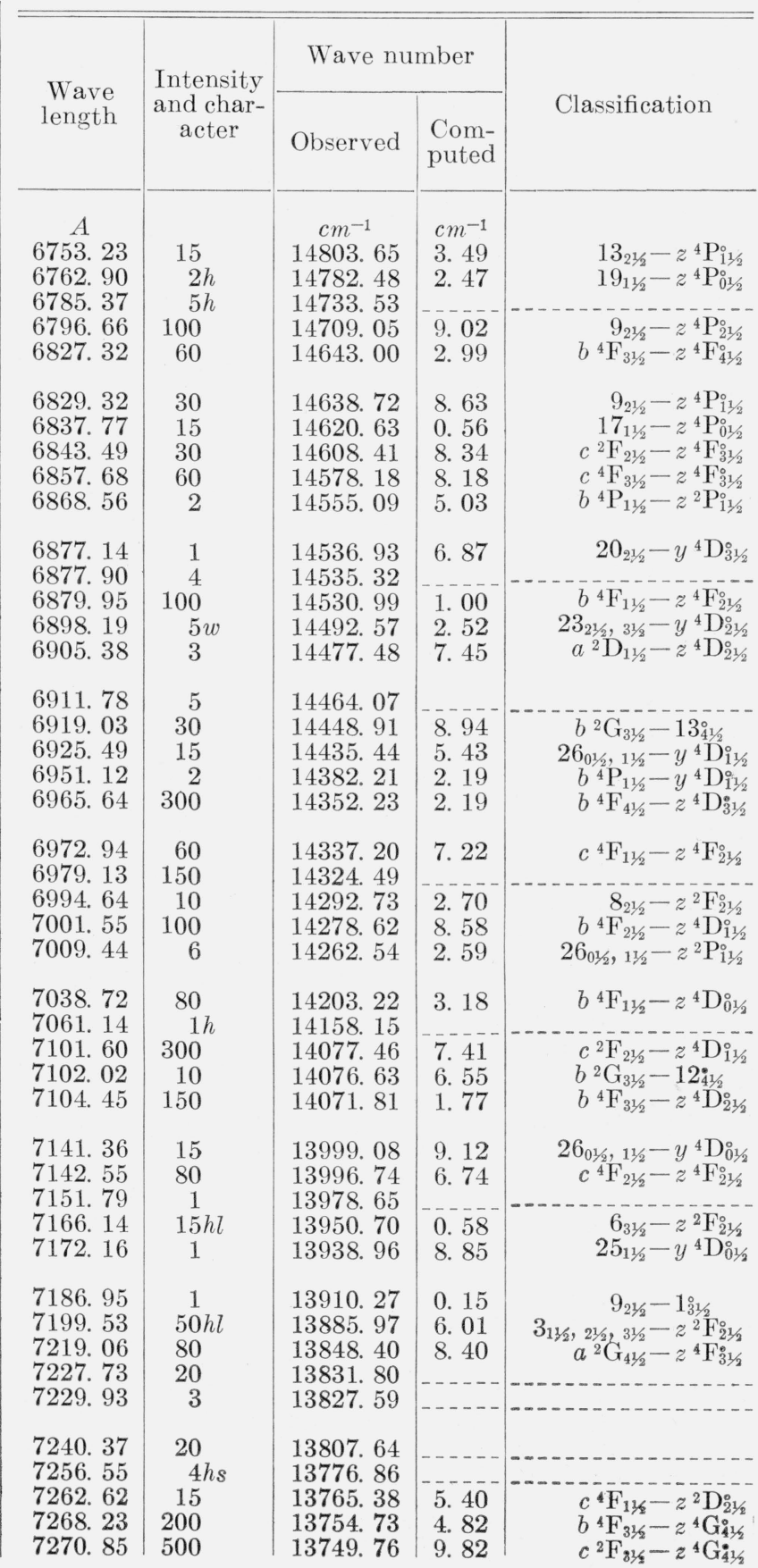


TABLE 1. Extension of the first spectrum of rhodium, Rh I-Continued

\begin{tabular}{|c|c|c|c|c|c|c|c|c|c|}
\hline \multirow{2}{*}{$\begin{array}{l}\text { Wave } \\
\text { length }\end{array}$} & \multirow{2}{*}{$\begin{array}{l}\text { Intensity } \\
\text { and char- } \\
\text { acter }\end{array}$} & \multicolumn{2}{|c|}{ Wave number } & \multirow{2}{*}{ Classification } & \multirow{2}{*}{$\begin{array}{l}\text { Wave } \\
\text { length }\end{array}$} & \multirow{2}{*}{$\begin{array}{l}\text { Intensity } \\
\text { and char- } \\
\quad \text { acter }\end{array}$} & \multicolumn{2}{|c|}{ Wave number } & \multirow{2}{*}{ Classification } \\
\hline & & Observed & $\begin{array}{l}\text { Com- } \\
\text { puted }\end{array}$ & & & & Observed & $\begin{array}{l}\text { Com- } \\
\text { puted }\end{array}$ & \\
\hline $\begin{array}{c}A \\
7271.93 \\
7273.01 \\
7273.61 \\
7282.83 \\
7285.22\end{array}$ & $\begin{array}{r}200 \\
60 \\
20 \\
20 \\
2\end{array}$ & $\begin{array}{c}\mathrm{cm}^{-1} \\
13747.72 \\
13745.67 \\
13744.54 \\
13727.15 \\
13722.64\end{array}$ & $\begin{array}{l}\mathrm{cm}^{-1} \\
\text { 7. } 65 \\
\text { 5. } 62 \\
\text { 4. } 45 \\
-.-- \\
-\cdots\end{array}$ & \begin{tabular}{c}
$b^{4} \mathrm{~F}_{21 / 2}-z^{4} \mathrm{~F}_{31 / 2}^{o}$ \\
$a^{2} \mathrm{G}_{31 / 2}-z^{4} \mathrm{~F}_{31 / 2}^{\circ}$ \\
$25_{11 / 2}-4_{11 / 2}^{\circ}$ \\
\hdashline
\end{tabular} & $\begin{array}{c}A \\
7981.97 \\
8016.60 \\
8029.93 \\
8036.09 \\
8041.60\end{array}$ & $\begin{array}{r}20 \\
80 \\
400 \\
250 \\
1 ?\end{array}$ & $\begin{array}{c}\mathrm{cm}^{-1} \\
12524.79 \\
12470.69 \\
12449.99 \\
12440.45 \\
12431.92\end{array}$ & $\begin{array}{r}c m^{-1} \\
4.75 \\
0.67 \\
50.00 \\
0.51 \\
1.98\end{array}$ & $\begin{array}{c}a^{2} \mathrm{G}_{41 / 2}-z^{4} \mathrm{G}_{41 / 2}^{\circ} \\
c^{2} \mathrm{~F}_{21 / 2}-z^{2} \mathrm{~F}_{31 / 2}^{\circ} \\
c^{4} \mathrm{~F}_{41 / 2}-z^{4} \mathrm{~F}_{41 / 2}^{\circ} \\
c^{4} \mathrm{~F}_{31 / 2}-z^{2} \mathrm{~F}_{31 / 2}^{\circ} \\
25_{11 / 2}^{\circ}-9_{21 / 2}^{\circ}\end{array}$ \\
\hline $\begin{array}{l}7290.51 \\
7293.22 \\
7299.80 \\
7314.93 \\
7330.13\end{array}$ & $\begin{array}{r}5 \\
10 \\
20 \\
2 \\
5\end{array}$ & $\begin{array}{l}13712.69 \\
13707.59 \\
13695.23 \\
13666.91 \\
13638.57\end{array}$ & $\begin{array}{l}\text { 2. } 69 \\
-\overline{5} . \overline{17} \\
6.91 \\
-\cdots\end{array}$ & $\begin{array}{c}b^{4} \mathrm{P}_{11 / 2}-z^{2} \mathrm{P}_{01 / 2}^{\circ} \\
25_{11 / 2}-y^{2} \mathrm{D}_{21 / 2}^{\circ} \\
26_{01 / 2}, 11 / 2-5_{01 / 2}^{\circ}\end{array}$ & $\begin{array}{l}8043.92 \\
8045.36 \\
8048.01 \\
8053.29 \\
8058.97\end{array}$ & $\begin{array}{l}80 \\
400 \\
1 h ? \\
4 h \\
2 h\end{array}$ & $\begin{array}{l}12428.33 \\
12426.11 \\
12422.02 \\
12413.88 \\
12405.13\end{array}$ & $\begin{array}{l}\text { 8. } 36 \\
6.17 \\
\text { 1. } 97 \\
\text { 3. } 85 \\
5.16\end{array}$ & $\begin{array}{c}c^{2} \mathrm{~F}_{21 / 2}-z^{2} \mathrm{D}_{21 / 2}^{\circ} \\
c^{2} \mathrm{~F}_{31 / 2}-z^{4} \mathrm{~F}_{31 / 2}^{\circ} \\
a^{2} \mathrm{G}_{31 / 2}-z^{4} \mathrm{G}_{41 / 2}^{\circ} \\
19_{11 / 2}-y^{4} \mathrm{D}_{11 / 2}^{\circ} \\
24_{11 / 2}-x^{2} \mathrm{P}_{11 / 2}^{\circ}\end{array}$ \\
\hline $\begin{array}{l}7334.80 \\
7375.54 \\
7386.63 \\
7391.70 \\
7423.63\end{array}$ & $\begin{array}{r}1 \\
100 \\
40 \\
1 \\
20\end{array}$ & $\begin{array}{l}\text { 13629. } 89 \\
13554.60 \\
13534.26 \\
13524.97 \\
13466.80\end{array}$ & $\begin{array}{l}4.57 \\
4.29 \\
-6.72\end{array}$ & $\begin{array}{r}a^{2} \mathrm{D}_{21 / 2}-z^{4} \mathrm{D}_{31 / 2}^{\circ} \\
c^{4} \mathrm{~F}_{11 / 2}-z^{4} \mathrm{~F}_{11 / 2}^{1} \\
19_{11 / 2}-y{ }^{4} \mathrm{D}_{21 / 2}^{\circ}\end{array}$ & $\begin{array}{l}8063.50 \\
8077.71 \\
8078.42 \\
8136.18 \\
8159.71\end{array}$ & $\begin{array}{c}80 \\
8 \\
5 \\
80 h \\
4\end{array}$ & $\begin{array}{l}\text { 12398. } 16 \\
12376.35 \\
12375.26 \\
12287.41 \\
12251.97\end{array}$ & \begin{tabular}{l}
8.20 \\
\hdashline 7.39 \\
1.94
\end{tabular} & $\begin{array}{r}c^{4} \mathrm{~F}_{31 / 2}-z^{2} \mathrm{D}_{21 / 2}^{8} \\
b^{4} \mathrm{~F}_{31 / 2}-z^{4} \mathrm{D}_{31 / 2}^{\circ} \\
17_{11 / 2}-y^{4} \mathrm{D}_{11 / 2}^{\circ}\end{array}$ \\
\hline $\begin{array}{l}7430.79 \\
7442.39 \\
7446.76 \\
7449.33 \\
7453.42\end{array}$ & $\begin{array}{c}60 \\
300 \\
80 \\
10 h \\
2\end{array}$ & $\begin{array}{l}13453.82 \\
13432.85 \\
13424.97 \\
13420.35 \\
13412.97\end{array}$ & $\begin{array}{l}\text { 3. } 77 \\
\text { 2. } 87 \\
\text { 4. } 92 \\
\text { 0. } 25 \\
\text { 2. } 92\end{array}$ & $\begin{array}{r}b^{4} \mathrm{~F}_{11 / 2}-z^{4} \mathrm{D}_{11 / 2}^{\circ} \\
c^{2} \mathrm{~F}_{31 / 2}-z^{4} \mathrm{D}_{21 / 2}^{\circ} \\
c^{4} \mathrm{~F}_{21 / 2}-z^{2} \mathrm{D}_{21 / 2}^{\circ} \\
13_{21 / 2}-y{ }^{4} \mathrm{D}_{31 / 2}^{\circ} \\
a^{2} \mathrm{G}_{41 / 2}-z^{4} \mathrm{~F}_{41 / 2}^{\circ 1 / 2}\end{array}$ & $\begin{array}{l}8163.22 \\
8166.99 \\
8173.41 \\
8193.67 \\
8210.43\end{array}$ & $\begin{array}{l}1 \\
2 h \\
4 \\
60 h \\
10\end{array}$ & $\begin{array}{l}\text { 12246. } 71 \\
12241.05 \\
12231.44 \\
12201.19 \\
12176.28\end{array}$ & $\begin{array}{l}\text { 1. } 01 \\
\text { 1. } 36 \\
\text { 1. } 20\end{array}$ & $\begin{array}{r}19_{11 / 2}-z^{2} \mathrm{P}_{11 / 2}^{\circ} \\
c^{2} \mathrm{~F}_{21 / 2}-z^{4} \mathrm{G}_{21 / 2}^{\circ} \\
c^{4} \mathrm{~F}_{31 / 2}-z^{4} \mathrm{G}_{21 / 2}^{\circ}\end{array}$ \\
\hline $\begin{array}{l}7468.28 \\
7472.4 \\
7475.71 \\
7484.53 \\
7487.34\end{array}$ & $\begin{array}{c}5 h \\
1 ? \\
300 \\
1 h \\
1\end{array}$ & $\begin{array}{l}\text { 13386. } 29 \\
13379.19 \\
13372.98 \\
13357.23 \\
13352.21\end{array}$ & 2.93 & $\begin{array}{r}c^{2} \mathrm{~F}_{21 / 2}-z^{4} \mathrm{G}_{31 / 2}^{0} \\
-\ldots \ldots\end{array}$ & $\begin{array}{l}8276.25 \\
8310.38 \\
8321.65 \mathrm{z} \\
8369.67 \\
8389.69\end{array}$ & $\begin{array}{l}2 H l \\
2 h \\
3 \\
30 \\
6\end{array}$ & $\begin{array}{l}12077.45 \\
12029.84 \\
12013.55 \\
11944.61 \\
11916.12\end{array}$ & $\begin{array}{l}3.57 \\
4.68 \\
6.14\end{array}$ & $\begin{array}{l}b{ }^{4} \mathrm{P}_{11 / 2}-z^{4} \mathrm{P}_{01 / 2}^{\circ} \\
c^{4} \mathrm{~F}_{11 / 2}-z^{2} \mathrm{D}_{11 / 3}^{0} \\
b{ }^{4} \mathrm{~F}_{11 / 2}-z^{4} \mathrm{D}_{21 / 2}^{\circ}\end{array}$ \\
\hline $\begin{array}{l}7492.64 \\
7495.22 \\
7496.92 \\
7505.09 \\
7513.95\end{array}$ & $\begin{array}{r}40 \\
300 \\
40 \\
1 \\
80\end{array}$ & $\begin{array}{l}13342.72 \\
13338.17 \\
13335.15 \\
13320.63 \\
13304.92\end{array}$ & \begin{tabular}{c}
2.77 \\
8.17 \\
$-\cdots$ \\
\hdashline 4.81
\end{tabular} & $\begin{array}{r}c^{4} \mathrm{~F}_{31 / 2}-z^{4} \mathrm{G}_{31 / 2}^{\circ} \\
c^{4} \mathrm{~F}_{41 / 2}-z^{4} \mathrm{G}_{41 / 2}^{\circ} \\
17_{11 / 2}-y^{4} \mathrm{D}_{21 / 2}^{\circ}\end{array}$ & $\begin{array}{l}8422.23 \\
8425.59 \\
8465.17 \\
8503.16 \\
8509.52\end{array}$ & $\begin{array}{r}2 \\
100 \\
1 \\
1 h \\
2 h\end{array}$ & $\begin{array}{l}\text { 11870. } 08 \\
11865.35 \\
11809.87 \\
11757.11 \\
11748.32\end{array}$ & $\begin{array}{l}0.07 \\
5.38 \\
9.80 \\
8.31\end{array}$ & $\begin{array}{r}26_{01 / 2}, 11 / 2-y{ }^{2} \mathrm{P}_{11 / 2}^{\circ} \\
c^{4} \mathrm{~F}_{11 / 2}-z^{2} \mathrm{P}_{21 / 2}^{o} \\
25_{11 / 2}-y^{2} \mathrm{P}_{11 / 2}^{o} \\
-b{ }^{4} \mathrm{P}_{11 / 2}-z^{4} \mathrm{P}_{11 / 2}^{\circ}\end{array}$ \\
\hline $\begin{array}{l}7524.30 \\
7542.01 \\
7549.58 \\
7557.70 \\
7558.84\end{array}$ & $\begin{array}{r}20 \\
100 \\
8 \\
150 \\
3 h\end{array}$ & $\begin{array}{l}13286.62 \\
13255.42 \\
13242.13 \\
13227.90 \\
13225.91\end{array}$ & $\begin{array}{l}\text { 6. } 52 \\
5.39 \\
\text { 2. } 17 \\
\text { 7. } 92 \\
----\end{array}$ & $\begin{array}{r}25_{11 / 2}-6_{21 / 2}^{\circ} \\
9_{21 / 2}^{\circ}-y^{4} \mathrm{D}_{31 / 2}^{\circ} \\
26_{01 / 2}, 11 / 2-x^{2} \mathrm{P}_{11 / 2}^{\circ} \\
c^{4} \mathrm{~F}_{21 / 2}-z^{4} \mathrm{G}_{21 / 2}^{\circ} \\
-\end{array}$ & $\begin{array}{l}8547.29 \\
8560.83 \\
8588.66 \\
8615.21 \\
8630.23\end{array}$ & $\begin{array}{c}2 \\
5 \\
1 h \\
10 h \\
1\end{array}$ & $\begin{array}{l}\text { 11696. } 40 \\
11677.91 \\
11640.10 \\
11604.19 \\
11584.00\end{array}$ & $\begin{array}{l}7.92 \\
4.20 \\
-\end{array}$ & $\begin{array}{r}b{ }^{4} \mathrm{P}_{11 / 2}-z{ }^{4} \mathrm{P}_{21 / 2}^{o} \\
c^{4} \mathrm{~F}_{21 / 2}-z^{2} \mathrm{D}_{11 / 2}^{o}\end{array}$ \\
\hline $\begin{array}{l}7577.26 \\
7579.58 \\
7595.43 \\
7616.01 \\
7639.22\end{array}$ & $\begin{array}{r}150 \\
40 \\
1 \\
1 \\
10\end{array}$ & $\begin{array}{l}\text { 13193. } 75 \\
13189.72 \\
13162.20 \\
13126.63 \\
13086.75\end{array}$ & $\begin{array}{l}3.81 \\
-2.11 \\
6.62 \\
6.77\end{array}$ & $\begin{array}{r}c^{4} \mathrm{~F}_{21 / 2}-z^{4} \mathrm{~F}_{11 / 2}^{\circ} \\
24_{11 / 2}-y^{4} \mathrm{D}_{01 / 2}^{0} \\
10_{11 / 2},{ }^{21 / 2}-y^{4} \mathrm{D}_{21 / 2}^{\circ} \\
a^{2} \mathrm{G}_{41 / 2}-z^{4} \mathrm{G}_{51 / 2}^{\circ}\end{array}$ & $\begin{array}{l}8674.46 \\
8745.89 \\
8769.08 \\
8886.25 \\
8909.71\end{array}$ & $\begin{array}{c}5 h \\
3 \\
10 \\
1 ? \\
1\end{array}$ & $\begin{array}{l}11524.93 \\
11430.80 \\
11400.58 \\
11250.24 \\
11220.63\end{array}$ & $\begin{array}{l}\text { 4. } 90 \\
0.77 \\
0.61 \\
0.24 \\
0.59\end{array}$ & $\begin{array}{c}c^{4} \mathrm{~F}_{21 / 2}-z^{2} \mathrm{~F}_{21 / 2}^{\circ} \\
c^{2} \mathrm{~F}_{21 / 2}-z^{2} \mathrm{G}_{31 / 2}^{\circ} \\
c^{4} \mathrm{~F}_{31 / 2}-z^{2} \mathrm{G}_{31 / 2}^{\circ} \\
b^{2} \mathrm{G}_{31 / 2}-6_{21 / 2}^{\circ} \\
19_{11 / 2}^{\circ}-x^{2} \mathrm{P}_{11 / 2}^{\circ}\end{array}$ \\
\hline $\begin{array}{l}7641.18 \\
7690.10 \\
7707.97 \\
7736.91 \\
7757.00\end{array}$ & $\begin{array}{c}2 \\
200 \\
20 \\
4 h \\
2 h\end{array}$ & $\begin{array}{l}13083.39 \\
13000.16 \\
12970.02 \\
12921.51 \\
12888.04\end{array}$ & $\begin{array}{l}\text { 3. } 35 \\
0.18 \\
0.02 \\
1.44 \\
\text { 8. } 00\end{array}$ & $\begin{array}{c}19_{11 / 2}-z^{2} \mathrm{P}_{01 / 2}^{\circ} \\
c^{2} \mathrm{~F}_{21 / 2}-z^{4} \mathrm{~F}_{21 / 2}^{\circ} \\
c^{4} \mathrm{~F}_{31 / 2}-z^{4} \mathrm{~F}_{21 / 2}^{o} \\
17_{11 / 2}-z^{2} \mathrm{P}_{01 / 2}^{\circ} \\
25_{11 / 2}-y^{2} \mathrm{D}_{11 / 2}^{\circ}\end{array}$ & $\begin{array}{l}8933.51 \\
8935.37 \\
8986.74 \\
9040.20 \\
9041.37\end{array}$ & $\begin{array}{c}40 \\
3 \\
1 ? \\
3 \\
15\end{array}$ & $\begin{array}{l}\text { 11190. } 74 \\
11188.41 \\
11124.46 \\
11058.67 \\
11057.24\end{array}$ & \begin{tabular}{c}
0.76 \\
\hdashline 8.68 \\
\hdashline .32
\end{tabular} & 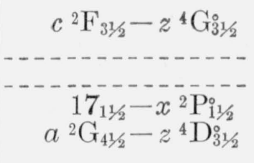 \\
\hline $\begin{array}{l}7769.85 \\
7772.92 \\
7773.48 \\
7791.65 \\
7824.92\end{array}$ & $\begin{array}{r}10 \\
300 \\
10 \\
400 \\
800\end{array}$ & $\begin{array}{l}12866.72 \\
12861.64 \\
12860.72 \\
12830.73 \\
12776.08\end{array}$ & $\begin{array}{l}1.65 \\
0.74 \\
6.15\end{array}$ & $\begin{array}{c}c^{2} \mathrm{~F}_{31 / 2}-z^{4} \mathrm{~F}_{41 / 2}^{\circ} \\
c^{4} \mathbf{F}_{31 / 2}-z^{2} \mathrm{G}_{41 / 2}^{\circ} \\
c^{4} \mathrm{~F}_{41 / 2}^{\circ}-z^{4} \mathrm{G}_{51 / 2}^{\circ}\end{array}$ & $\begin{array}{l}9058.26 \\
9083.47 \\
9102.10 \\
9124.46 \\
9126.13\end{array}$ & $\begin{array}{c}6 h \\
1 h \\
3 \\
10 h \\
5\end{array}$ & $\begin{array}{l}11036.62 \\
11005.99 \\
10983.46 \\
10956.55 \\
10954.54\end{array}$ & $\begin{array}{l}6.57 \\
4.54\end{array}$ & $b^{4} \mathrm{~F}_{21 / 2}-z^{4} \mathrm{D}_{31 / 2}^{\circ}$ \\
\hline $\begin{array}{l}7830.04 \\
7846.52 \\
7847.74 \\
7871.60 \\
7899.49\end{array}$ & $\begin{array}{r}250 \\
100 \\
8 \\
2 h \\
2 h\end{array}$ & $\begin{array}{l}12767.84 \\
12741.01 \\
12738.95 \\
12700.41 \\
12655.57\end{array}$ & $\begin{array}{l}0.95 \\
8.92 \\
0.43 \\
5.52\end{array}$ & $\begin{array}{c}b^{4} \mathrm{~F}_{21 / 1}-z^{4} \mathrm{D}_{21 / 2}^{\circ} \\
a^{2} \mathrm{G}_{31 / 2}-z^{4} \mathrm{D}_{21 / 2}^{\circ} \\
b^{2} \mathrm{G}_{31 / 3}-x^{2} \mathrm{~F}_{21 / 2}^{\circ} \\
20_{21 / 2}-4_{11 / 2}^{\circ}\end{array}$ & $\begin{array}{l}9149.85 \\
9177.12 \\
9221.28 \\
9231.48 \\
9246.92\end{array}$ & $\begin{array}{l}2 h \\
2 h \\
1 \\
1 h \\
2 h\end{array}$ & $\begin{array}{l}10926.12 \\
10893.68 \\
10841.50 \\
10829.53 \\
10811.57\end{array}$ & \begin{tabular}{c}
6.15 \\
\hdashline 1.59 \\
\hdashline 1.49
\end{tabular} & $\begin{array}{r}1_{31 / 2}-y^{4} \mathrm{D}_{31 / 2}^{8} \\
-b^{2} \mathrm{G}_{313}-y^{2} \mathrm{D}_{21 / 2} \\
9_{21 \leq 4}-x^{2} \mathrm{P}_{11 / 2}\end{array}$ \\
\hline
\end{tabular}


TABLE 1. Extension of the first spectrum of rhodium, Rh I-Continued

\begin{tabular}{|c|c|c|c|c|c|c|c|c|c|}
\hline \multirow{2}{*}{$\begin{array}{l}\text { Wave } \\
\text { length }\end{array}$} & \multirow{2}{*}{$\begin{array}{l}\text { Intensity } \\
\text { and char- } \\
\text { acter }\end{array}$} & \multicolumn{2}{|c|}{ Wave number } & \multirow{2}{*}{ Classification } & \multirow{2}{*}{$\begin{array}{l}\text { Wave } \\
\text { length }\end{array}$} & \multirow{2}{*}{$\begin{array}{c}\text { Intensity } \\
\text { and char- } \\
\text { acter }\end{array}$} & \multicolumn{2}{|c|}{ Wave number } & \multirow{2}{*}{ Classification } \\
\hline & & Observed & $\begin{array}{l}\text { Com- } \\
\text { puted }\end{array}$ & & & & Observed & $\begin{array}{l}\text { Com- } \\
\text { puted }\end{array}$ & \\
\hline$A$ & & $c m^{-1}$ & $\mathrm{~cm}^{-1}$ & & $A$ & & $\mathrm{~cm}^{-1}$ & $\mathrm{~cm}^{-1}$ & \\
\hline & $1 h$ & & 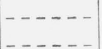 & $\ldots$ & 9713.84 & 1 & 10291.77 & & D \\
\hline & $6 h$ & $\begin{array}{l}10718.17 \\
10678.65\end{array}$ & $87 \overline{3}$ & $\cdots$ & 9716.97 & 15 & 10288.45 & 8. 50 & $c^{2} \mathrm{~F}_{31 / 2}-z^{2} \mathrm{~F}_{31 / 2}^{o 1}$ \\
\hline $\begin{array}{l}9361.91 \\
9424.55\end{array}$ & $2 h$ & & $\begin{array}{l}8.73 \\
7.64\end{array}$ & $c^{2} \mathrm{~F}_{31 / 2}-z^{2} \mathrm{G}_{41 / 2}^{\circ}$ & 9748.87 & $2 h$ & 10254. 79 & 4. 78 & $9_{21 / 2}-8_{21 / 2}^{\circ}$ \\
\hline & $\begin{array}{l}50 \\
1 h\end{array}$ & $\begin{array}{l}10607.68 \\
10572.49\end{array}$ & 7.64 & $c^{2} \mathrm{~F}_{21 / 2}-z^{2} \mathrm{D}_{11 / 2}^{\circ}$ & $\begin{array}{l}9757.11 \\
9761.40\end{array}$ & 150 & 10246. 12 & 6. 19 & $c^{2} \mathrm{~F}_{31 / 2}-z^{2} \mathrm{D}_{21 / 2}^{\circ}$ \\
\hline & & & & - n. & 9801.40 & 1 & 10241.03 & - - - - & 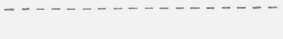 \\
\hline 9477.57 & $2 h$ & 10548. 34 & & $2 \mathrm{~T}_{\mathrm{N}}-2 \mathrm{~F}_{0}$ & 9790. 70 & $2 h$ & 10210.98 & 1. 11 & $b{ }^{4} \mathrm{P}_{11 / 2}-z^{2} \mathrm{D}_{11 / 2}^{\circ}$ \\
\hline 9495.56 & 20 & 10528. 36 & 8. 34 & $c^{2} \mathrm{~F}_{21 / 2}-z^{2} \mathrm{~F}_{21 / 2}^{\circ}$ & 9846.07 & $1 h$ & 10153.56 & $-\cdots+$ & - n \\
\hline $\begin{array}{l}9522.88 \\
9536.60\end{array}$ & $\begin{array}{l}6 \\
3 h\end{array}$ & $\begin{array}{l}10498.14 \\
10483.04\end{array}$ & 8. 18 & $c^{4} \mathrm{~F}_{31 / 2}-z^{2} \mathrm{~F}_{21 / 2}^{\circ}$ & 9850.93 & $\begin{array}{l}3 h \\
2 h\end{array}$ & $\begin{array}{l}10148.55 \\
10112.37\end{array}$ & & $\cdots$ \\
\hline 9541.31 & 5 & 10477.86 & 7.87 & $c^{4} \mathrm{~F}_{11 / 2}-z^{4} \mathrm{P}_{21 / 2}^{0}$ & $\begin{array}{l}9886.17 \\
9948.38\end{array}$ & $\begin{array}{l}2 h \\
80\end{array}$ & $\begin{array}{l}10112.37 \\
10049.14\end{array}$ & 9. 19 & $c^{2} \mathrm{~F}_{31 / 2}-z^{4} \mathrm{G}_{21 / 2}^{\circ}$ \\
\hline 9578.82 & 1 & 10436. 84 & & 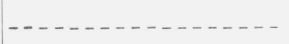 & 9959.61 & 3 & 10037. 80 & & \\
\hline 9580.54 & $1 ?$ & 10434. 96 & --- & - & 10015. 42 & $2 h$ & 9981. 87 & 1. 80 & $25_{11 / 2}-x^{2} \mathrm{D}_{11 / 2}^{\circ}$ \\
\hline 9597. 94 & 1 ? & 10416. 04 & 748 & $\ldots \ldots+\cdots$ & 10082. 38 & $1 h$ & 9915.58 & $-\cdots$ & 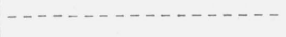 \\
\hline $\begin{array}{l}9605.86 \\
9613.84\end{array}$ & $\begin{array}{c}20 \\
2 h\end{array}$ & $\begin{array}{l}10407.46 \\
10398.82\end{array}$ & $\begin{array}{l}\text { 7. } 48 \\
----\end{array}$ & $\begin{array}{c}c^{4} \mathrm{~F}_{11 / 2}-z^{4} \mathrm{P}_{11 / 2}^{1} \\
-\end{array}$ & $\begin{array}{l}10119.00 \\
10121.96\end{array}$ & $\begin{array}{l}2 h \\
3 h\end{array}$ & $\begin{array}{l}9879.71 \\
9876.80\end{array}$ & 6.85 & $c^{4} \mathrm{~F}_{41 / 2}-z^{2} \mathrm{~F}_{31 / 2}^{\circ}$ \\
\hline 9620.75 & 1 & 10391. 35 & & $\ldots$ & 10188. 37 & 1 & 9812.44 & & - \\
\hline 9626.53 & $2 h$ & 10385. 12 & & - - - - & 10499. 29 & $1 W H ?$ & 9521.83 & & $\ldots$ \\
\hline 9632.58 & $1 h$ & 10378. 59 & & - & 10809. 46 & $1 h$ & 9248.62 & 8. 60 & $c^{2} \mathrm{~F}_{31 / 2}-z^{2} \mathrm{G}_{31 / 2}^{\circ}$ \\
\hline 9671.76 & $2 h$ & 10336.55 & & - - & 11021. 74 & $1 h$ & 9070.48 & 0.44 & $c^{2} \mathrm{~F}_{21 / 2}-z^{4} \mathrm{P}_{11 / 2}^{\circ}$ \\
\hline
\end{tabular}

Although others have published data on $\mathrm{RhI}$ extending to $8615 \mathrm{~A}$, a comparison of the intensities of corresponding lines shows that the present estimates are on a more open scale that is approximately five times greater than that used in earlier publications. In addition, new lines have been discovered in the regions previously investigated. Of the 238 new wavelength measurements here listed, 148 had previously been measured by Meggers [2], of which 111 were included by Molnar and Hitchcock [6]. With the aid of a grating ruled with 15,000 lines/in., it has been possible to increase the precision of some RhI wavelengths. With the new photographic emulsions it has been possible to investigate this spectrum out to $12000 \mathrm{~A}$, although there is only one $\mathrm{Rh}$ I line beyond $11000 \mathrm{~A}$.

One of the main difficulties encountered in the spectral observation of the platinum metals in open air ares is the presence of many atomic oxygen and nitrogen lines, and unidentified molecular lines, particularly between 8300 and $11000 \mathrm{~A}$. A comparison with this Bureau's wave length data, published and unpublished, shows that table 1 contains no lines originating from atmospheric constituents in the atomic state. Atmospheric molecular lines were more troublesome, and it was a problem to determine which lines could be legitimately discarded as such. Fortunately, a photograph of the i idium spectrum in the same region was available, and all lines appearing in both spectra were ascribed to atmospheric spectra and removed from the $\mathrm{Rh}$ I list. Several calcium, sodium, potassium, and copper lines were also present in the rhodium spectra, but the first three would be expected in almost any metallic sample. The copper lines were introduced when the arc jumped to the copper electrode holder.

\subsection{Classification of Observed Lines of Rh I}

With the term values as given by Molnar and Hitchcock [6], a systematic subtraction between even and odd levels with differences between 8000 and $17000 \mathrm{~cm}^{-1}$ was carried out. This served to classify 35 lines belonging to known terms. In addition, a search for all the known term separations among three dozen of the stronger lines still remaining unclassified has resulted in the discovery of two new even levels. With these two new levels, one at $23655.93 \mathrm{~cm}^{-1}$, suspected to be a $b{ }^{4} P_{13 / 2}$ level, and the other at $27876.52 \mathrm{~cm}^{-1}$, suspected to be a $b^{2} G_{31 / 2}$ level, it was possible to classify 18 more lines of RhI. One of these lines had previously been incorrectly classified. This is seen in the following example by the much closer agreement between the observed and computed vacuum wave numbers given by the new $b^{2} G_{3 \frac{1}{2}}$ level:

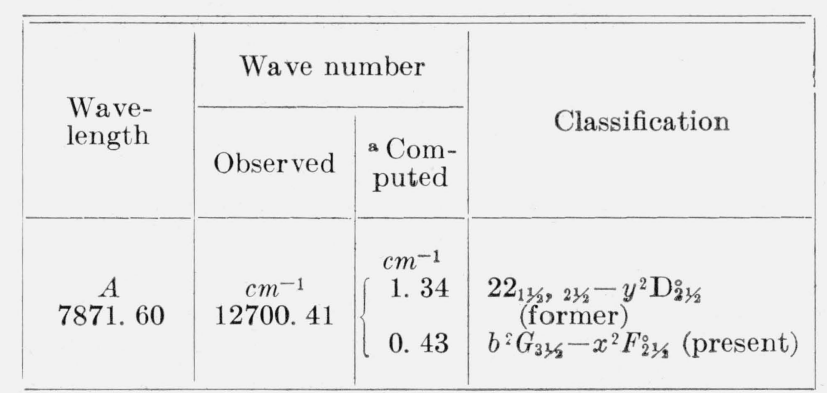

a Only the last 3 digits are given here and in table 1 for the computed wave number.

The new transitions in the infrared have resulted in the assignment of $J$-values to two known levels for which the values were previously ambiguous. 
The level $20_{11 / 2,21 / 2}$ at $51324.35 \mathrm{~cm}^{-1}$ combines with levels having $\vec{J}$-values of $1 \frac{1}{2}$ and $3 \frac{1}{2}$, which, according to the transition rule of $\Delta J=0, \pm 1$, determines the $J$-value to be $2 \frac{1}{2}$. In like manner, the level $24_{11 / 2,21 / 3}$ at $51636.54 \mathrm{~cm}^{-1}$ combines with levels of $J=0 \frac{1}{2}, 1 \frac{1}{2}$, and $2 \frac{1}{2}$, and has therefore been assigned $J=1 \frac{1}{2}$.

A comparison of the observed and computed wave numbers for classified lines shows that the differences average $0.04 \mathrm{~cm}^{-1}$, with 30 percent of the lines agreeing to within $0.03 \mathrm{~cm}^{-1}$. These differences are within the error of measurement for long waves.

The author takes pleasure in thanking William F. Meggers, Chief of the Spectroscopy Section, for assigning this project, and for his continued interest and guidance.

\section{References}

[1] C. E. Moore, Atomic energy levels as derived from the analyses of optical spectra, NBS Circ. 467, III (in preparation).

[2] W. F. Meggers, Sci. Pap. BS 20, 31 (1925) S499.

[3] C. P. Snyder, Astrophys. J. 14, 179 (1901).

[4] W. F. Meggers and C. C. Kiess, J. Opt. Soc. Am. Rev. Sci. Instr. 12, 438 (1926).

[5] L. A. Sommer, Z. Physik 45, 147 (1927).

[6] J. P. Molnar and W. J. Hitcheock, J. Opt. Soc. Am. 30, 523 (1940).

[7] W. F. Meggers and R. J. Murphy, J. Research NBS 48, 334 (1952) RP2320.

[8] W. F. Meggers and K. Burns, Sci. Pap. BS 18, 191 (1922) S441.

[9] S. M. Burka, J. Franklin Inst. 189, 25 (1920).

[10] Trans. Intern. Astron. Union, 5, 84 (1935).

Washington, July 1952. 